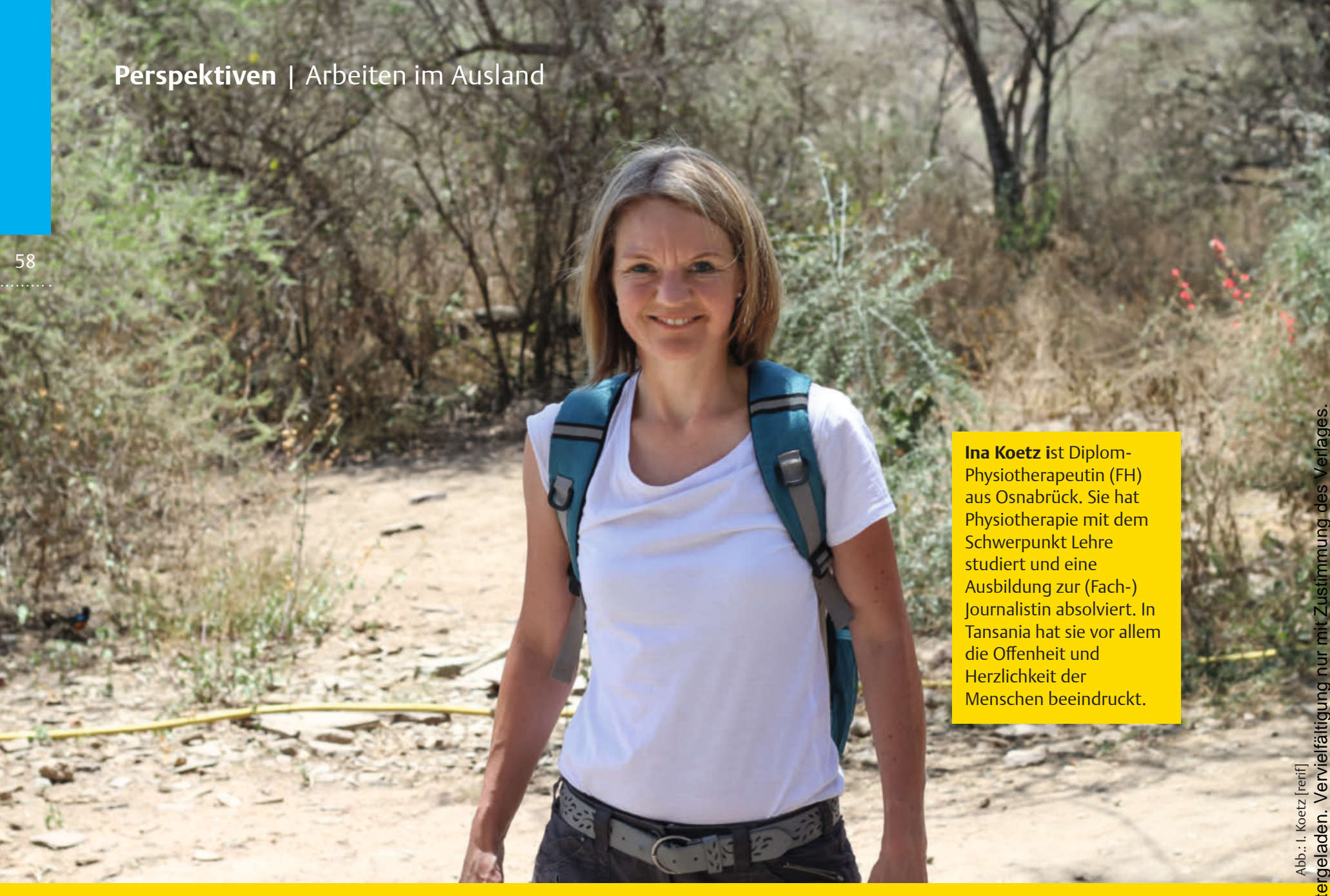

\title{
Willkommene Knochendoktoren
}

Als Physiotherapeutin in Tansania In Tansania sind Physiotherapeuten „Daktari wa mifupa“, die Ärzte für Knochen. Für drei Monate nimmt sich Ina Maria Koetz eine Auszeit, um dort in einem Krankenhaus zu arbeiten. Dass Therapeuten hier Röntgenbilder auswerten, Frakturen versorgen und sogar Medikamente verschreiben, ist für sie Neuland.

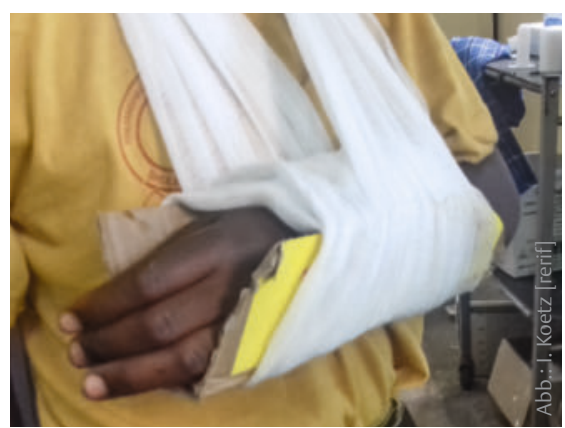

ABB.1 Notdürftige Versorgung: Die Fraktur eines jungen Mannes haben Familienmitglieder provisorisch mit einem Stück Karton stabilisiert.

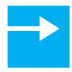

Endlich ist es so weit. Mit zwei Koffern und einem Rucksack stehe ich abends um 20 Uhr am Kilimandjaro Airport in Arusha. „Karibu, welcome to Tanzania“, begrüßt mich eine junge afrikanische Frau. Der Flughafen ist überraschend klein. Ich fülle einige Formulare aus und gebe meine Fingerabdrücke ab. Dann gibt mir die Frau mein Visum inklusive erhoffter Arbeitserlaubnis. „Wenn ich nun noch abgeholt werde, ist alles perfekt", denke ich und entdecke einen Herrn, der freudestrahlend ein Schild mit meinem Namen hochhält.

Das Abenteuer hat begonnen: drei Monate als Physiotherapeutin im St. Elizabeth Hospital in Tansania, einem der ärmsten Länder der Welt und fünftgrößtes Land Afrikas.

\section{Betten für jährlich 55.000 Patienten}

$\rightarrow$ Das St. Elizabeth Hospital ist das zweitgrößte Krankenhaus der Region und befindet sich am Fuße des Kilimandscharo, mitten im MassaiGebiet. Als ursprüngliche Apotheke ist es im Laufe seiner fast 40-jährigen Geschichte stetig gewachsen und versorgt jährlich etwa 55.000 Patienten. Dafür stehen 100 Betten zur Verfügung. Durch Spenden und auf Initiative eines deutschen Arztes hin konnten 2015 eine Kinderstation und 2016 eine Augen- und eine 


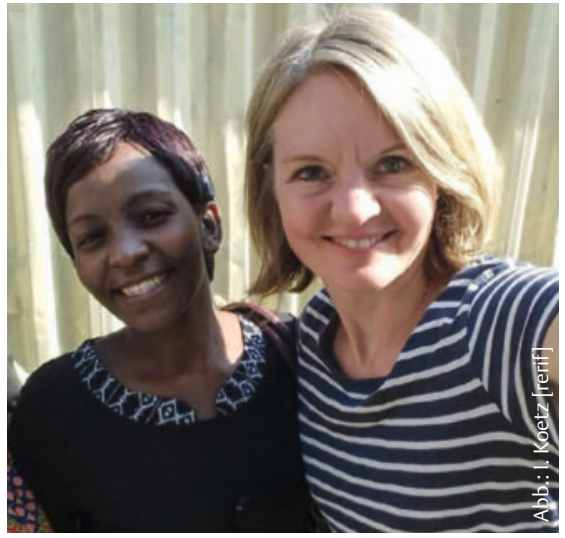

ABB. 6 Mit ihrer Arbeitskollegin Happy hatte Ina Maria Koetz jede Menge Spaß.

Hektik und Zeitdruck sucht man vergeblich $\rightarrow$ Jeder Morgen beginnt mit einem Gottesdienst für alle Mitarbeiter. Anschließend findet der sogenannte Morning Report statt, an dem auch ich teilnahm. Vertreter aller Fachbereiche besprechen die wichtigsten Fälle und Vorkommnisse der Nacht.

Wenn der Therapiebetrieb um neun Uhr startet, sitzen bereits einige Patienten vor dem Behandlungsraum. Termine gibt es nicht. Warten bedeutet für die Menschen nichts Unangenehmes. Hektik gibt es nicht, was manchmal erstaunlich und eine Herausforderung für deutsche Gewohnheiten ist. Selbst Patienten mit frischen Frakturen warten geduldig auf die Versorgung. Lediglich Patienten mit offenen Frakturen behandeln Ärzte bevorzugt und schnell.

Es ist erstaunlich, wie beherrscht Kinder ihre Verletzungen ertragen. Selbst wenn der Therapeut eine Fraktur reponiert, verbergen sie ihren Schmerz. Therapeuten verschreiben bei Bedarf auch Schmerzmittel.

Hands-on findet selten statt $\rightarrow$ Insgesamt unterscheidet sich die Arbeitsweise der Therapeuten in dieser Klinik sehr von der in deutschen Kliniken. Sie bezieht sich auf den orthopädischchirurgischen Bereich, wobei Therapeuten auch internistisch und neurologisch sehr gut ausgebildet sein müssen. Oft sind sie die erste Anlaufstelle für Patienten, und nach einer Befunderhebung entscheiden sie, ob Rückenschmerzen die Folge einer Tuberkuloseinfektion, der Thoraxschmerz auch ein Herzinfarkt und die Fazialisparese die Folge eines Schlaganfalls sein könnten.

Eine große Herausforderung ist die Aufklärungsarbeit. Viele Kinder kommen mit einer
Pneumonie in die Klinik. Den Eltern ist oft nicht bewusst, dass der Rauch der Kochstelle, den die auf dem Rücken fixierten Kinder oft stundenlang einatmen, gesundheitsschädlich ist. Und richtig schwierig wird es, wenn Patienten den Kräften der noch häufig praktizierenden „Wunderheiler“ glauben und alles andere ablehnen.

Behandlungsdauer ist nicht festgelegt $\rightarrow$ Da Patienten eine Übungsbehandlung kaum akzeptieren, findet keine Anleitung zu Eigenübungen statt. Eine schnelle Versorgung mit Medikamenten und Gipsverbänden bestimmt den Alltag. Dies ist nachvollziehbar, da die oft körperlich hart arbeitenden Tansanier nur wenig Energie für anstrengende körperliche Übungen aufbringen können.

Die therapeutische Behandlung besteht überwiegend in der Befunderhebung und Erstversorgung. Nach Entfernung des Gipsverbandes gibt es meist keine Nachbehandlung. Eine eingeschränkte Beweglichkeit der Gelenke wird toleriert. Nur selten kommt ein Patient häufiger als zweimal zur Therapie.

Angenehm ist, dass Therapeuten keine vorgegebenen Zeitfenster haben. Jeder Patient bekommt die Aufmerksamkeit, die er braucht. An Hilfsmitteln hingegen fehlt es: Es gibt weder Unterarmgehstützen noch Rollstühle oder Rollatoren. Nach Therabändern, Pezzibällen oder anderen Trainingsgeräten sucht man vergeblich.

Mit Unistudium zum Bachelor oder Master $\rightarrow$ Wer in Tansania eine Stelle als Therapeut sucht, hat gute Chancen, nach kurzer Zeit eine zu finden. Es gibt eine Art Tariflohn für Anstellungen in staatlichen Krankenhäusern, der bei etwa einer Million TZS liegt, was 370 Euro entspricht. Gibt es keinen Tariflohn, verdienen sie deutlich weniger, zwischen 180 und 250 Euro.

Die Ausbildung setzt das A-Level voraus, was dem deutschen Abitur entspricht. Sie findet an der Uni statt und schließt nach vier Jahren mit dem Bachelor-Grad ab. Die Kosten für das Studium belaufen sich auf rund 1.500 Euro pro Jahr, was sich nicht jeder leisten kann. Im ersten Jahr findet nur theoretischer Unterricht statt, in Mikrobiologie, Pharmakologie, Radiologie, Kinderpflege und Ethik. Im zweiten Jahr wer- den Mikrobiologie und Radiologie vertieft. Das Reponieren von Gelenken und Frakturen steht ebenso auf dem Stundenplan wie Soziologie, Ökonomie und die in Deutschland bekannten physiotherapeutischen Techniken wie PNF, Manuelle Therapie usw. Das zweite Jahr, in das ein sechswöchiges pädiatrisches Praktikum integriert ist, schließt mit einer Zwischenprüfung ab. Im dritten Jahr beschäftigen sich die Studenten vormittags mit wissenschaftlichem Arbeiten und bereiten die Themen ihrer Bachelorarbeit vor. Nachmittags findet in den letzten beiden Jahren ein Praktikum in einem Krankenhaus statt.

Englisch wird oft nicht beherrscht $\rightarrow$ Trotz der finanziellen Not vieler Tansanier ist ihre Herzlichkeit überwältigend. Tansania ist ein sehr friedliches Land ohne Schießereien oder Bombenanschläge. Die verschiedenen Stämme leben in dem christlich geprägten Land friedlich zusammen. Die Herzlichkeit und Offenheit zeigen sich in der Klinik und bei den Menschen auf der Straße. „Karibu“, was auf Deutsch „Herzlich willkommen“ bedeutet, schallt aus allen Winkeln der Stadt.

Die Tansanier sind sehr interessiert an den „Mzungu“, den weißen Menschen. Die Kommunikation ist nicht immer einfach. Englisch ist zwar offizielle Landessprache, wird aber oft nicht beherrscht. Für viele ist Kisuaheli Muttersprache. Nach ein bisschen Übung klappt auch die Kommunikation darin. Für mich steht fest, dass dies nicht mein letzter Aufenthalt in Tansania war.

Ina Maria Koetz

\section{Spenden}

\section{Jeder Euro hilft}

\section{Möchten Sie das St. Elizabeth Hospital in Arusha, Tansania, mit einer Spende unterstützen?}

\section{Spendenkonto:}

Eine-Welt-Ausschuss St. Mauritius Stichwort: Ina für Elisabeth Hospital Arusha IBAN DE78 403510600003000700 Kreissparkasse Steinfurt 